\title{
Real Time Volumetric Ultrasound Imaging System
}

\author{
Olaf T. von Ramm and Stephen W. Smith
}

\begin{abstract}
A real time volumetric ultrasound imaging system has been developed for medical diagnosis. The scanner produces images analogous to an optical camera and supplies more information than conventional sonograms. Potential medical applications include improved anatomic visualization, tumor focalization, and better assessment of cardiac function. The system uses pulse-echo phased array principles to steer a two-dimensional array transducer of 289 elements in a pyramidal scan format. Parallel processing in the receive mode produces 4992 scan lines at a rate of approximately 8 frames/second. Echo data for the scanned volume is presented as projection images with depth perspective, stereoscopic pairs, multiple tomographic images, or C-mode scans. (C) 1990 by W.B. Saunders Company.
\end{abstract}

KEY WORDS: diagnostic ultrasound, phased array. three-dimensional, volumetric, diagnostic imaging, real time.

A HIGH-SPEED volumetric ultrasound imaging system that uses pulse-echo principles analogous to sonar and radar has been developed for medical application. Conventional ultrasound cross-sectional imaging has become a valuable diagnostic modality in virtually every medical speciality. ${ }^{1}$ In routine clinical practice, ultrasound pulse-echo images of soft tissue are obtained with high spatial resolution (1 to $2 \mathrm{~mm}$ ), at real time, video frame rates $(30 / \mathrm{sec})$ using portable systems. Currently, the most sophisticated medical ultrasound scanners use a phased linear array of approximately 100 piezoelectric transducer elements to steer and focus the ultrasonic beam along approximately 150 directions in a sector scan format. Beam steering and focusing is accomplished by properly delaying the transmit pulses and the echoes returning to the transducer array from tissue structures within the body. ${ }^{2}$

Over the years, there has been interest in the medical imaging community in constructing three-dimensional (3D) renderings from serial cross-sectional images obtained via magnetic resonance imaging, $x$-ray computed tomography and diagnostic ultrasound. ${ }^{3}$ The advantages of such renderings include more realistic anatomic visualization and improved localization and sizing of mass lesions for surgical procedures. Current techniques are limited by lengthy data acquisition times, computer processing times, and extensive operator interaction that preclude imaging of dynamic structures without sophisticated gating techniques.

In the field of diagnostic ultrasound, 3D imagings systems have been proposed in the past. However, these have been too slow for clinical applications ${ }^{4,5}$ or have operated primarily in a through-transmission mode requiring a complex water-path apparatus. ${ }^{6,7}$ Our objective was to develop an ultrasound system, which uses a hand-held transducer, capable of scanning a volume rapidly and enabling the 3-D visualization of dynamic structures in real time.

In order to extend cross-sectional ultrasound imaging to volumetric imaging, three advances were necessary: (1) a hand-held transducer was needed that could be steered rapidly and focused at any desired orientation in the volume; (2) the data acquisition rate had to be increased significantly to obtain image data from many planes in real time; (3) a display method was required, which allowed the visualization of all or part of the echo data obtained from the scanned tissue volume.

In order to demonstrate expeditiously the feasibility of high-speed volumetric ultrasound imaging, the extant Duke University phased array research scanner ${ }^{8}$ was appropriately modified. Figure 1 illustrates the concept of a volumetric phased array ultrasound imaging system that uses a two-dimensional (2D) array of transducer elements to produce a pyramidal scan composed of eight steering directions in the azimuth dimension, $\Theta$, by 16 steering directions in the elevation dimension, $\Phi$.

From the Department of Biomedical Engineering, Duke University, Durham, NC; the Center for Devices and Radiological Health, Food \& Drug Administration, Rockville, MD; and the Department of Radiology, Duke University, Durham, NC.

Supported in part by Health and Human Sciences Grant No. NCI CA37586, National Science Foundation/Engineering Research Center Grant No. CDR-86222, and by the Food and Drug Administration.

Address reprint requests to Olaf T. von Ramm, Department of Biomedical Engineering, Duke University, Durham, NC 27706.

(0) 1990 by W.B. Saunders Company.

0897-1889/90/0304-0002\$03.00/0 


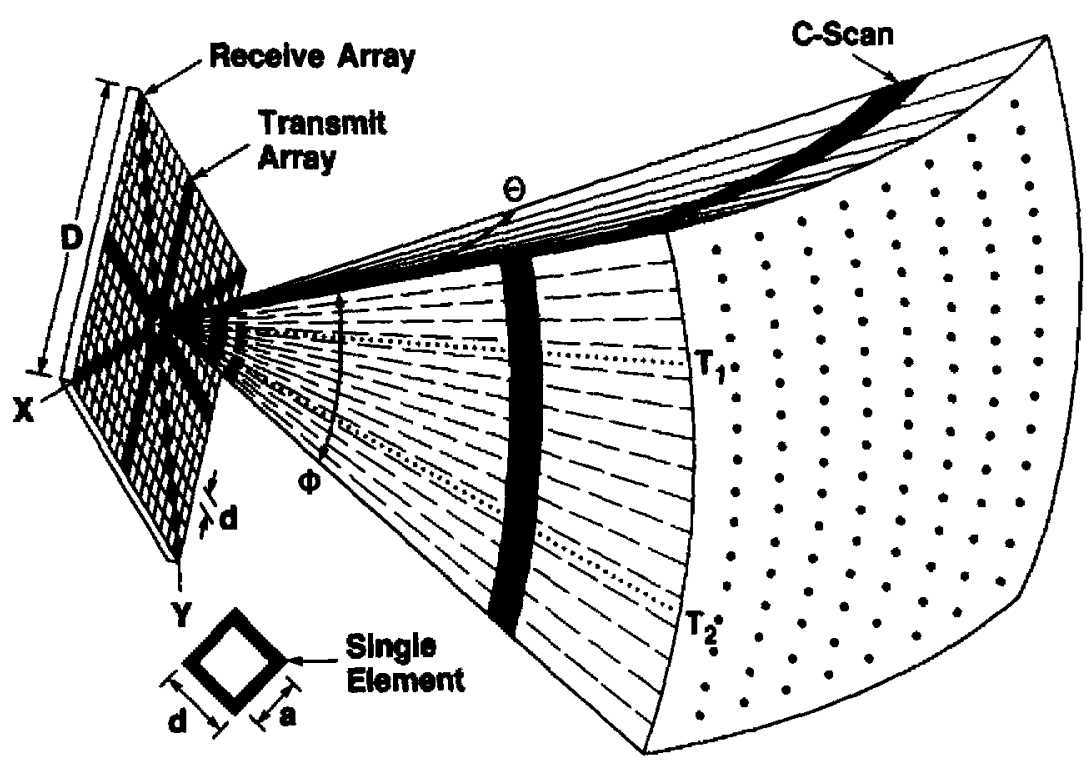

Fig 1. Pyramidal san format of 3D ultrasound imaging system showing 2D phesed array trensducer, and the transmit and receive elements within the array. Transmit directions are indicated by dotted lines. receive directions by dashes. Dote at end of scan volume are image points used to produce the perspective display of Fig 3. Adjustable C-scan plane is indicated by darkened region.
In order to obtain best image quality, all of the $17 \times 17$ elements of the array should be used. However, the Duke system is limited to 32 independent transmit channels and 32 independent receive channels. In this study, the Mills cross antenna design of radio astronomy ${ }^{9}$ was chosen for the transmit aperture (shown by the solid cross in Fig 1) and an independent Mills cross for the receive transducer aperture (shown by the shaded diamonds of Fig 1) to provide adequate sampling of the $2 \mathrm{D}$ array. The two crosses are positioned at $45^{\circ}$ with respect to each other and neither one utilizes the center element. This design enabled the imaging system to be steered and focused to any desired angle with a small number of phased array channels while maintaining adequate spatial resolution for highcontrast targets. The tradeoff associated with this transducer design increases the undesired off-axis energy from the transducer.

The 2D array of 289 transducer elements illustrated in Fig 1 was fabricated in our laboratory with independent transmit and receive crosses of 32 elements each. The array operates at 1.7 $\mathrm{MHz}$ with inter-element spacing of $0.64 \mathrm{~mm}$ yielding a spatial resolution of $\simeq 4^{\circ}$. The phased array time delays were calculated using the Pythagorean theorem for each transducer array element at each transmit and receive focal point for each line in the pyramidal scan.

Conventional pulse-echo data acquisition is limited by the round trip propagation time of an ultrasound pulse into the body that is determined by the velocity of sound in tissue, $\simeq 1540 \mathrm{~m} / \mathrm{sec}$. The data acquisition was increased through parallel signal processing in the receive mode in which eight steering directions were obtained simultaneously for each transmitted ultrasound pulse.

This parallel processing technique, which we have described previously, ${ }^{10}$ is illustrated in Fig 1 for a few representative lines. In the $\Phi$ direction, the orientation of the two transmit pulses $T_{1}$ and $T_{2}$ (produced by the transmit array) are shown by the dotted lines. By using the smaller transmit cross array, the transmit beam is broadened to a width of $\simeq 6^{\circ}$. On each side of each transmit beam, four receive mode scan lines spaced at $0.5^{\circ}$ increments (shown by the dashed lines) are obtained in parallel by the receive mode array. The steering directions are shown on an exaggerated scale in the figure.

The receive mode parallel processing is accomplished by the addition of small independent tapped delay lines to the principle electronic delay lines used for steering and focusing. These small delays add the desired steering angle increment in the receive mode of the phased array system. The eight to one parallel processing increases the data acquisition rate of the echo signals by a factor of eight. Thus, in the volumetric scanner 12 transmitted pulses steered in the elevation ( $\Phi$ ) direction over a $65^{\circ}$ sector produce 96 steering directions. In the azimuth direction $(\Theta)$, no parallel processing is currently implemented, so that 52 transmit pulses yield 52 scan 
lines steered over a $65^{\circ}$ sector. Thus, in each pyramidal scan, 624 transmit pulses produced 4992 scan lines per image at a frame rate of 8 images/second. As a trade-off, the broadened transmit beam, necessary for the increased data acquisition rate of parallel processing, increases undesired off-axis energy from the transducer and degrades the image contrast by $\simeq 13 \mathrm{~dB}$ in the resulting 3D images.

Figure 2 is a block diagram of the high-speed volumetric ultrasound phased array scanner. The figure shows the PDP-11 computer that serves as the imaging system controller and provides the phased array delay data to the rest of the imaging system. Also shown are the 32 transmit pulsers that fire the transmit transducer array and the 32 preamplifiers that amplify the ultrasonic echoes from the receive mode array. The figure also shows the electronic delay lines that provide the receive mode delays for steering and focusing as well as providing the 8 to 1 receive mode parallel processing. Finally, the figure shows the video processing system and CRT scope for the display of the ultrasound images.

For viewing the ultrasound images, an optical holographic display ${ }^{11}$ or $3 \mathrm{D}$ vibrating mirror ${ }^{12}$ would be desirable, but these are not currently available as on-line, real time devices. We have implemented two different display techniques using a conventional 2D cathode-ray tube screen. In the first method, all the echo data along each scan line is displayed as a unique point in the image. Since the echo data is written on the screen continuously, the viewer's eye integrates the data to a single brightness value. This technique is illustrated in Fig 1 in which the $8 \times 16$ scan directions are represented by a matrix of $8 \times 16$ dots. In this way, the ultrasound scanner produces a projection image and functions as the direct analogue of a normal optical camera. The $(x, y)$ position of each point in the display is proportional to the tangent of the steering angle of the corresponding scan line $(\tan \Theta, \tan \Phi)$. In this display, a more distant object that subtends a smaller angle within the pyramidal scan is displayed as a smaller image. Thus depth is encoded in the display as in the human eye or a conventional optical camera.

Figure 3 shows preliminary results of our 3D imaging system in water tank evaluations of the projection display. Figure $3 \mathrm{~A}$ is a 4992-line pyramidal scan of a $14 \mathrm{~mm}(9 / 16 \mathrm{in})$ wrench in water positioned parallel to the transducer face at a depth of $4 \mathrm{~cm}$. Figure $3 \mathrm{~B}$ and $C$ illustrate the effect of perspective as the wrench is moved to 6 and $9 \mathrm{~cm}$, respectively, from the face of the transducer.

An interesting feature of all ultrasound scanning is the ability to suppress unwanted image data simply by gating the receive information or varying the receive gain. ${ }^{13}$ In this way, overlying structures can be peeled away in the projection display to create the so called $C$ scan. Such a range gated image from an object is illustrated in

Fig 2. Block diagram of high-speed 3D imaging system.

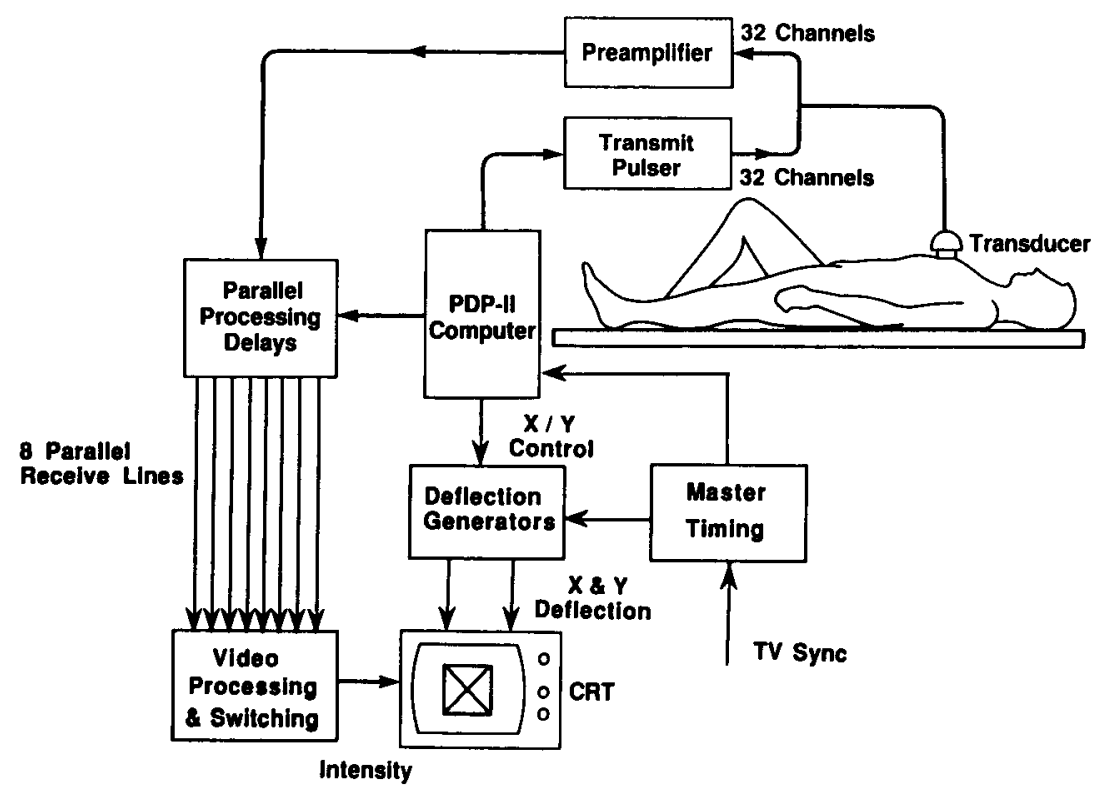



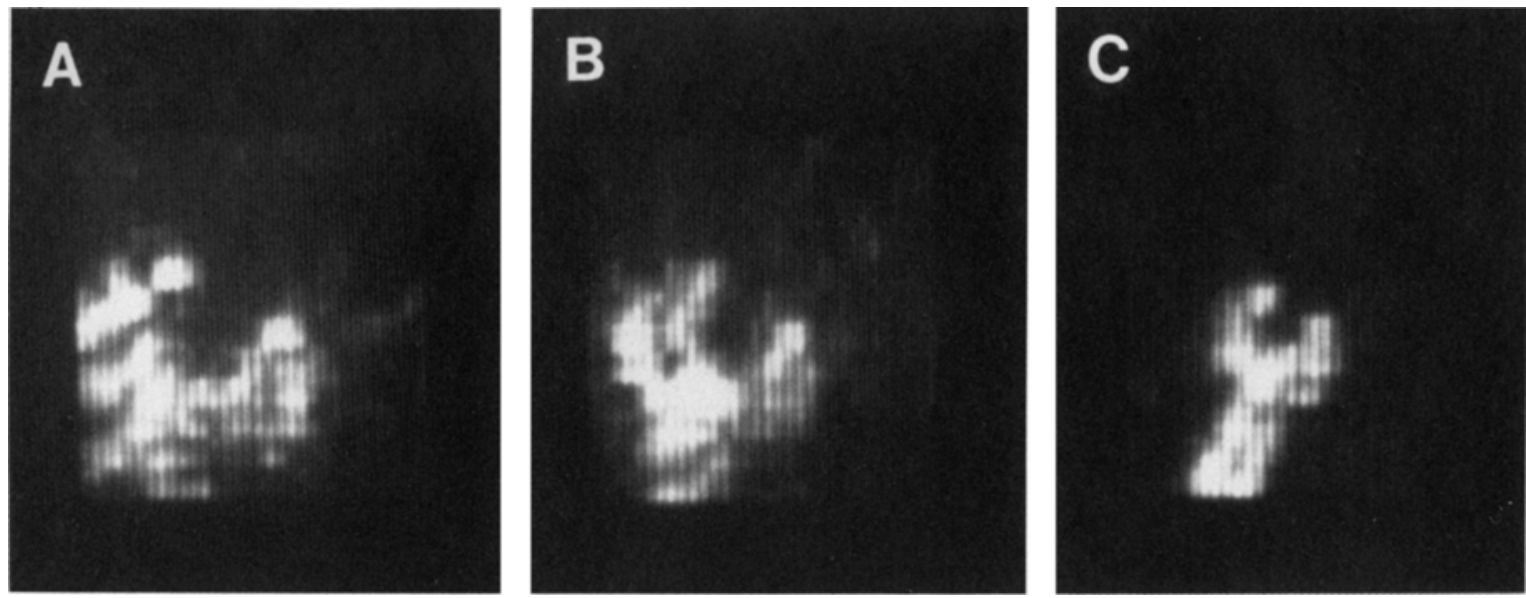

Fig 3. (A) Projection image with perspective of a wrench at a depth of $4 \mathrm{~cm}$ in water obtained with parallel processing and 4992 scan lines at 8 frames $/$ second. (B) Image of same wrench at depth of $6 \mathrm{~cm}$. (C) Wrench at $9 \mathrm{~cm}$.

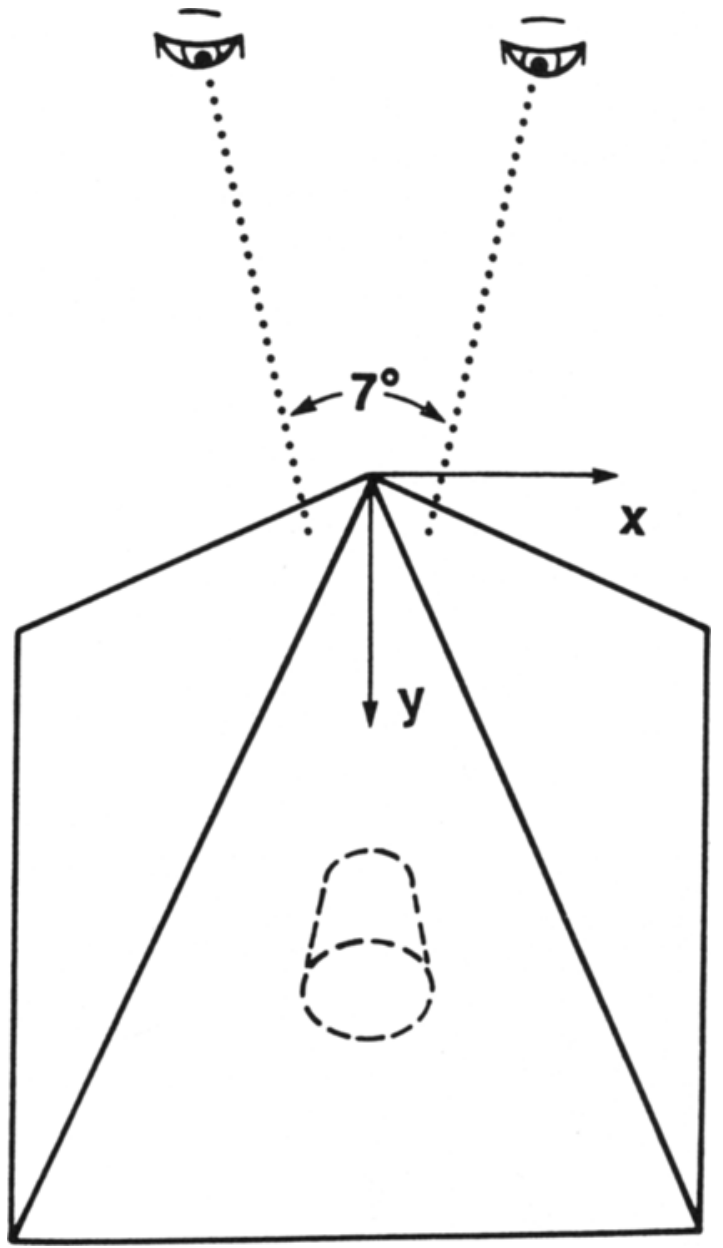

Fig 4. Stereoscopic pairs are produced simultaneously by viewing two planar projections separated by $7^{\circ}$ of objects within the pyramidal volume.
Fig 1 by the shaded slice parallel to the transducer face. $C$ scans that commonly require many seconds per image frame $\mathrm{e}^{14,15}$ can now be obtained at rates of 8 frames per second.

In the second display method, all of the echo data along each scan line is displayed by its projection on a plane passing through the apex of the pyramid. Viewed from the apex of the pyramidal scan, the image lines appear to radiate in all directions from a common origin. Lines steered at small angles are viewed as points, while lines at large steering angles are viewed along their entire length. The object can also be viewed from any chosen direction by simply projecting echo information into a plane orthogonal to that view direction. Since all the 3D echo data is available

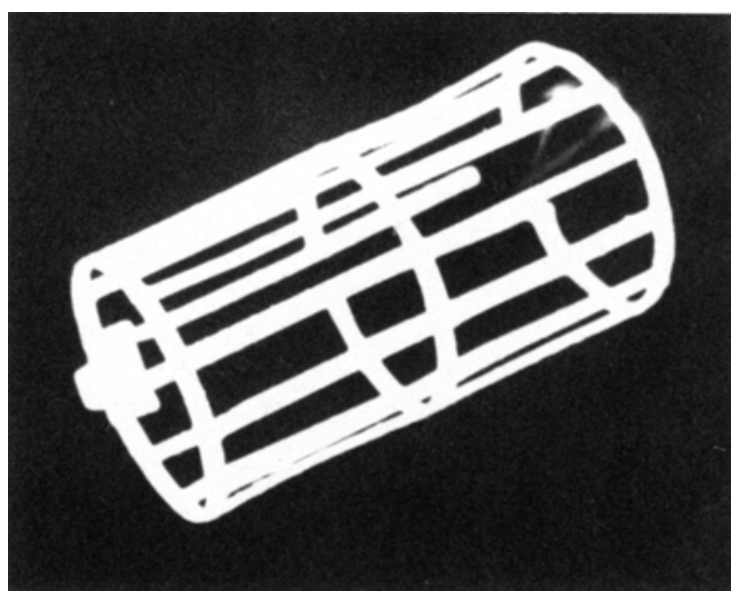

Fig 5. Optical photograph of cylindrical metal cage placed in scan volume. 
Fig 6. (A) Left eye view of 3D ultrasound projection image of metal cage of Fig 5. (B) Right eye view of ultrasound projection image of metal cage of Fig 5.
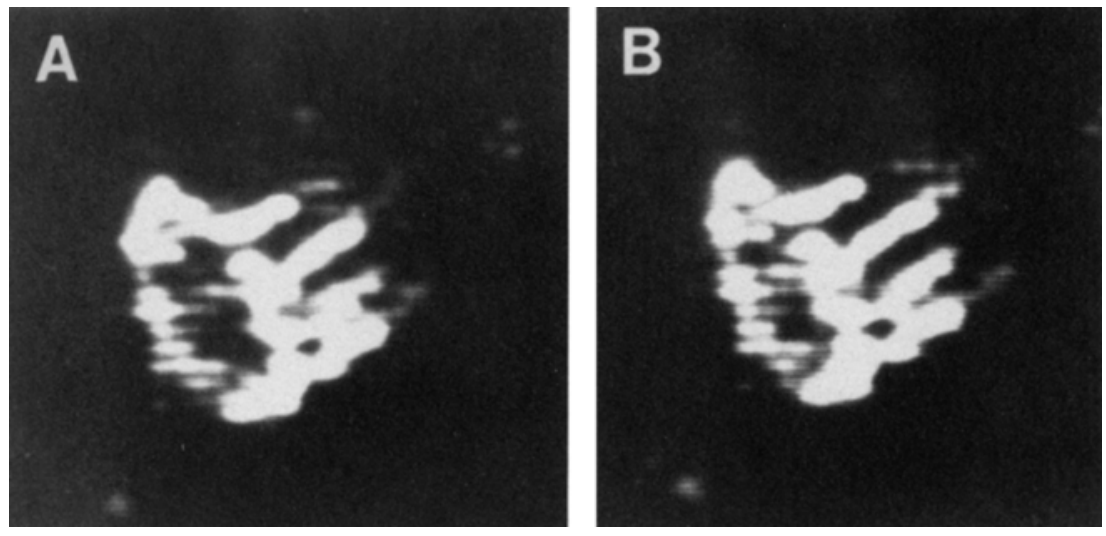

independent of the display method, the pyramidal scan can actually be displayed and viewed from two directions simultaneously. This then permits the display of simultaneous stereoscopic pairs. This display technique is illustrated in Fig 4 in which the apex of the pyramidal scan is centered between the two eyes of an observer. The image presented to each eye is the projection of echo data into planes rotated $\pm 3.5^{\circ}$ about the $x$ axis.

In a preliminary study, we have implemented such a stereoscopic display with the volumetric ultrasound scanner. A $65^{\circ}$ pyramidal scan composed of 576 lines $(24 \times 24)$ was obtained without parallel processing at a frame rate of approximately 8 frames per second. The scan was displayed on-line as viewed in coordinate systems rotated $+3.5^{\circ}$ and $-3.5^{\circ}$ about the $\mathrm{x}$-axis. These images are equivalent to those viewed by the left and right eyes respectively in Fig 4 . A cylindrical metal cage $(4.4 \mathrm{~cm} \times 8.9 \mathrm{~cm})$, shown in an optical photograph in Fig 5, was scanned perpendicular to its long axis. The left eye view and right eye view are shown in Figs $6 \mathrm{~A}$ and $\mathrm{B}$, respectively. When these images are fused by the observer, as a stereoscopic pair by viewing the images with the corresponding eyes, the 3D nature of the object is clearly visible. It should be emphasized that the stereoscopic images are not computer reconstructed images.

We have demonstrated the feasibility of on line, high speed 3D ultrasound imaging. Further improvements in image quality are still needed before clinical trials can determine the benefits of this new diagnostic technique. Phased array systems exceeding 200 independent transducer channels may be necessary to produce highquality images of low-contrast structures such as tissue parenchyma. Parallel processing certainly needs to be implemented in both steering dimensions, $\Theta$ and $\Phi$, to increase data acquisition rates by as much as a factor of 100 over conventional rates. These advances are achievable with current technology, especially with the development of customized large-scale integrated circuitry. ${ }^{16}$ Of equal importance is the further development of 3D display technology and studies of the human perception of 3D information. With these improvements, 3D real-time ultrasound images may then be a medical advance equivalent to the step from the one-dimensional ultrasound graphs of the 1950s to today's sonograms and will permit $3 \mathrm{D}$ ultrasound imaging of moving organs such as the heart.

\section{REFERENCES}

1. Popp RL, Macovski A: Ultrasonic diagnostic instruments. Science 210:268-273, 1980

2. von Ramm OT, Smith SW: Beam steering with linear arrays. IEEE Transaction Biomed Eng BME 30:438-452, 1983

3. McCann HA, McCann JC, Harp S, et al: Multidimensional ultrasonic imaging for cardiology. Proceedings IEEE, 76:1063-1073, 1988

4. Howry DH, Posakony GJ, Cushman CR, et al: Three- dimensional and stereoscopic observation of body structures by ultrasound. J Appl Physiol 9:304-306, 1956

5. Brown TG: Visualization of soft tissues in two and three dimensions-limitations and development. Ultrasonics 5:118124,1967

6. Thurstone FL: Proceedings of symposium on biomedical engineering, in Metherell AF, et al (eds) Acoustical Holography vol 1. New York, NY, Plenum, 1969

7. Green PS, Schaefer LF, Macovski A: Considerations 
for diagnostic ultrasonic imaging, in Wade $G$ (ed) Acoustical Holography (vol 4). New York, NY, Plenum, 1972, pp 97-111

8. von Ramm OT, Thurstone FL; Cardiac imaging using a phased array ultrasound system I: System design. Circulation $53: 258,1975$

9. Steinberg BD: Principles of Aperture and Array System Design. Wiley Sons, New York, NY, 1976

10. Shattuck DP, Weinshenker MD, Smith SW, et al: Explososcan: A parallel processing technique for high-speed ultrasound imaging with linear phased arrays. J Acoust Soc Amer 75:1273-1282, 1984

11. Baum G, Stroke GW: Optical holographic threedimensional ultrasonography. Science 189:994-995, 1975
12. Fuchs H, Pizer SM, Heinz ER, et al: Adding a true 3-D display to a raster graphic system. IEEE Transcripts. Computer Graphics and Applications 2:73-78, 1982

13. Wells PNT: Biomedical Ultrasonics. New York, NY, Academic, 147-148, 1977

14. McReady VR, Hill CR: A Constant Depth Ultrasonic Scanner. Brit J Rad 44:747-750, 1971

15. Northved A, Holm HH, Kristensen JK, et al: An ultrasonic spherical section scanner. Ultra Med Biol 1:183186,1974

16. O'Donnell M: Applications of VLSI circuits to medical imaging. Proceedings of the Institute of Electrical and Electronic Engineers 76:1 106-114, 1988 